\title{
FET-biosensor for cardiac troponin biomarker
}

\author{
Mohd Khairuddin Md Arshad ${ }^{1,2, *}$, Mohamad Faris Mohamad Fathil ${ }^{1}$, and Uda Hashim ${ }^{1.2}$ \\ ${ }^{1}$ Institute of Nano Electronic Engineering, Universiti Malaysia Perlis, 01000 Malaysia \\ ${ }^{2}$ School of Microelectronic Engineering, Universiti Malaysia Perlis, 02600 Malaysia
}

\begin{abstract}
Acute myocardial infarction or myocardial infarction (MI) is a major health problem, due to diminished flow of blood to the heart, leads to higher rates of mortality and morbidity. The most specific markers for cardiac injury are cardiac troponin I (cTnI) and cardiac troponin $\mathrm{T}(\mathrm{cTnT})$ which have been considered as 'gold standard'. Due to higher specificity, determination of the level of cardiac troponins became a predominant indicator for MI. Currently, field-effect transistor (FET)-based biosensors have been main interest to be implemented in portable sensors with the ultimate application in point-of-care testing (POCT). In this paper, we review on the FET-based biosensor based on its principle of operation, integration with nanomaterial, surface functionalization as well as immobilization, and the introduction of additional gate (for ambipolar conduction) on the device architecture for the detection of cardiac troponin I (cTnI) biomarker.
\end{abstract}

\section{Introduction}

Cardiac biomarkers are the indicators, which have been pre-dominantly used in the detection of acute myocardial infarction (AMI). It is logical to use protein quantification in a blood sample for this purpose as stated by Rosalki et al. [1]. The myocyte is the major cell in the heart, and the heart's purpose is to pump blood. When myocytes essentially cannot be regenerated due to heart cells die, then cardiac function has a high probability of being damaged. When the cell dies, the biomarker proteins (i.e. myoglobin, creatine-kinase MB, C-reactive protein and cardiac troponin are most commonly used) inside the cell will be released, with proteins in the cytoplasm leaving the cell more rapidly than the ones in membranes or fixed cell elements.

For MI, cardiac troponin $\mathrm{T}$ (cTnT) and cardiac troponin I (cTnI) are regarded as more sensitive and specific [2]. Both are released from the death cell within 2-4 $\mathrm{h}$ and 3-4 h, respectively, after the onset of MI symptoms [3]. In principle, cTnT and cTnI remain in the blood stream approximately more than 10 days, reaches to peak approximately 1-2 days [4] after myocardial injury. Because of its prolonged release in the blood, these biomarkers are useful in diagnosing sub-acute myocardial infarction [2]. In normal patients, the level of cTnI concentration is around $1 \mathrm{pg} / \mathrm{ml}$, but increased to 100 $\mathrm{ng} / \mathrm{ml}$ in MI patients [5]. Even the concentration as low as $10 \mathrm{pg} / \mathrm{ml}$ can be related to heart failure. Fig. 1 shows general information regarding $\mathrm{MI}$.

Biosensors can be used to detect and quantify the target molecules involved with cardiac biomarker interaction. Biosensors are integrated diagnostic devices, which merge biological or biologically-derived sensing element associated with a physicochemical transducer [6]. Generally, surface of a suitable transducer of a biosensor is immobilized with a biological receptor material (DNA, RNA or antibody), which enables conversion of biochemical signal into quantifiable electronic signal [7].

To overcome the limitations of conventional immunoassay-labelled method such as enzyme-linked immunosorbent assay (ELISA), which is lack of portability, late detection time, and high complexity of fabrication process [8], the electrical detection of biomolecular interaction development is highly beneficial because it is suitable to become the low cost portable sensor and can be used non-specialized personnel [9]. Electrical detection is conducted by transducing the molecular binding event into a usable electrical signal [10]. In order to sense chemical and biological species which is very small in size, researchers have intensely studied nanostructures, such as nanowires (NWs), nanobelts, carbon nanotubes (CNTs), graphene, and nanoparticles for biosensing, due to comparable size between sensor and target. The electrical detection has become the main interest in recent year, which was evidenced by higher number of publications related to diagnostic MI.

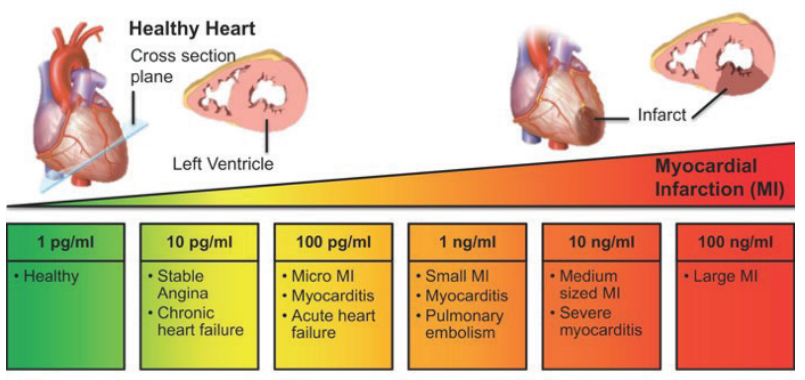

Fig. 1. Heart-related problem based on cTn concentration level [5].

* Corresponding author: mohd.khairuddin@unimap.edu.my 


\section{Field-effect transistors biosensor}

Field-effect transistor (FET)-based biosensors are currently at the centre of interest for biological molecules (biomolecules) detection due to anticipated qualities, i.e. label-free, fast electrical detection, low power operation, easy to move from one place to another, low cost for mass production, and both sensor and measurement system are compatible to be integrated into a single chip [11]. These kind of biosensor is a FET gated by biomolecules, which change the gate charge distribution, hence influencing the conductance of the FET channel [12].

\subsection{Principle of operation.}

The FET-based biosensor (Fig. 2) is a device that enables the detection of biomolecules by having surface's channel, which is directly exposed to the surrounding, producing difference surface potential on the surface of a transducer upon biological interaction, thus contributes to modulation of the current flow inside the semiconductor channel, located between drain and source regions [13] enabling biological recognition. The application of biomolecules to the gate electrode generates an electric field, which controls the current flow between source and drain region [14]. The type of semiconductor (n- or $\mathrm{p}$ type) determines the type of carriers (electrons or holes) of the conduction current.
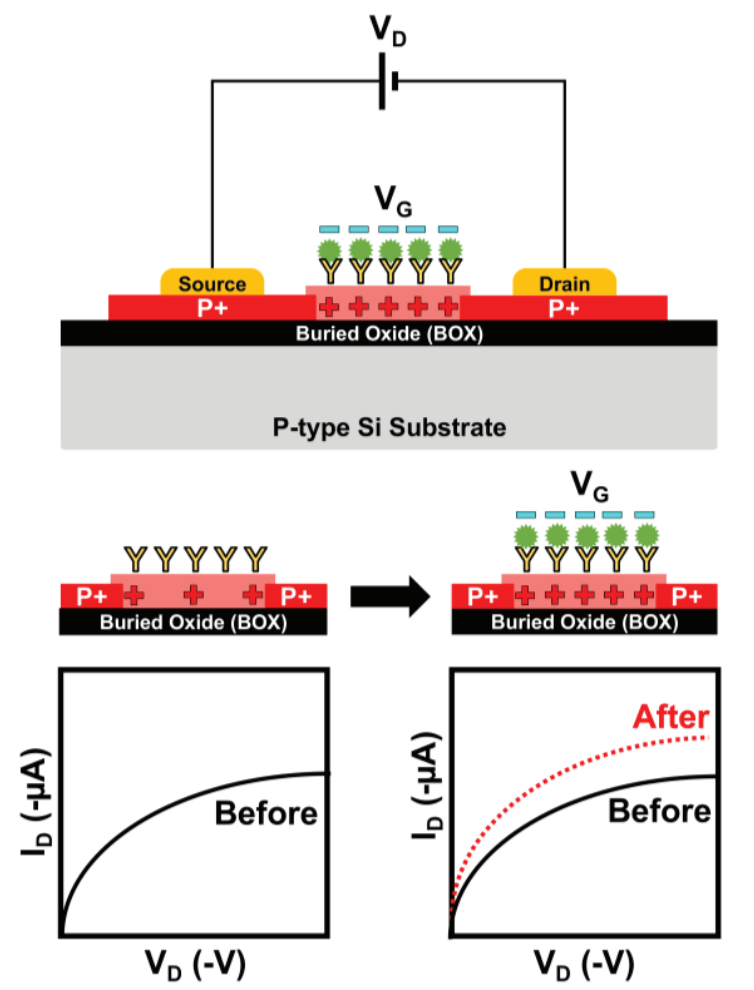

Fig. 2. SOI FET-based biosensor in term of device architecture and electrical characteristic [13].

\subsection{Integration with nanomaterial as transducer}

In the recent years, nanostructured metal-oxides (NMOs) materials (i.e. $\mathrm{ZnO}, \mathrm{TiO} 2, \mathrm{SnO} 2$, and etc.) have grabbed considerable attention as transducer in development of biosensor. They exhibited unique non-toxic nanomorphology with functional biocompatibility, and interesting catalytic properties [15]. The materials also offered appropriate biomolecules immobilization microenvironment by owning strong adsorption capability, hence improved electron transfer between the biomolecule's active site and electrode and enhanced biosensing characteristic [16]. NMOs also offer high surface area for higher biomolecules loading and help biomolecules to maintain its bioactivity by providing a suitable microenvironment [17]. The NMOs' unique characteristic provided possibility of 1) utilizing electronic signal transduction as interface for biomolecules detection and 2) next generation of novel bioelectronics devices [15]. Selection of an appropriate NMOs for immobilization of the required bio-receptor is important in fabrication of efficient biosensor. The biosensor's performance is also influenced by immobilized surface between NMOs bind with a biomolecule. This can be done by having an effective NMOs immobilized surface, which aid a biomolecule to maintain its activity with high stability by exhibiting a biocompatible microenvironment. Commonly, NMOs bind to the biomolecules by physical adsorption or covalent binding. Weak interactions of NMO to biomolecule (e.g. van der Waals, electrostatic, physisorption) are some examples of the physical adsorption, which rely on the surface morphology, reaction medium and net surface charge. On the other hand, covalent binding of NMO to a biomolecule relies on the available functional groups on the NMO's surface. This can be arranged through suitable chemical reactions. Therefore, the use of NMOs in development of biosensor can enhanced sensitivity and limit of detection, in addition to simple and inexpensive fabrication deposition process.

\subsection{Surface functionalization and immobilization}

The specific detection of biomolecules is possible by utilizing specific biological receptors (bio-receptors), i.e. DNA, RNA, aptamers, or Antibodies (Abs), which are immobilized at the surface of the transducer through covalent binding with suitable chemical linkers. The biomolecules, which can be positively or negatively charge, captured by the bio-receptors and influence the changes in electrical behaviour of the transducers. The changes, whether increase or decrease the conductance of the transducer's channel (depending on the n- or p-type of semiconductor materials), allow more or less current flows from source to drain region [11].

The covalent bonding as in Fig. 3 is the most frequently used technique for immobilization of cardiac biomarkers as bio-receptors in FET-based biosensor. This technique alters the biosensor's surface for acquiring a reactive functional group to enable attachment of the specific bio-receptor. Proteins comprised of amino acid side chain, which owned nucleophilic functional groups (i.e. amino, carboxylic, imidazole, thiol, hydroxyl, and etc.). These functional groups are used for the coupling, which requires the utilization of reagent such as glutaraldehyde (GA), carbodiimide, succinimide esters, 
maleinimides, or periodate for covalent immobilization [18]. The advantages of using covalent binding for bioreceptors' immobilization on the surface of the transducer is the enhancement in bio-receptors' uniformity, density, and distribution, in addition to the reproducibility and homogeneity of the surfaces [19]. This technique also possible to reduce or remove some common complications i.e. instability, diffusion and aggregation, or inactivation of biomolecules [19].

The high selectivity of a biosensor is possible through immobilization of the bio-receptor, which is the cTnI monoclonal antibody (Mab-cTnI), on the sensor to allow specific interaction of compounds [20]. Mab-cTnI is a sophisticated biomolecule, built from hundreds of singular amino acids arranged in highly ordered sequence and highly specific to cTnI biomarker [19]. It is produced by the immune system which identifies and remembers an cTnI biomarker that occupies the human body. The produced Mab-cTnI, commonly illustrated as $\mathrm{Y}$-shape, is a complementary match to the cTnI biomarker, which is identical to the lock-and-key mechanism of enzymesubstrate binding) [21], hence suitable to be utilized as exceptional bio-receptors in bio-sensing applications.

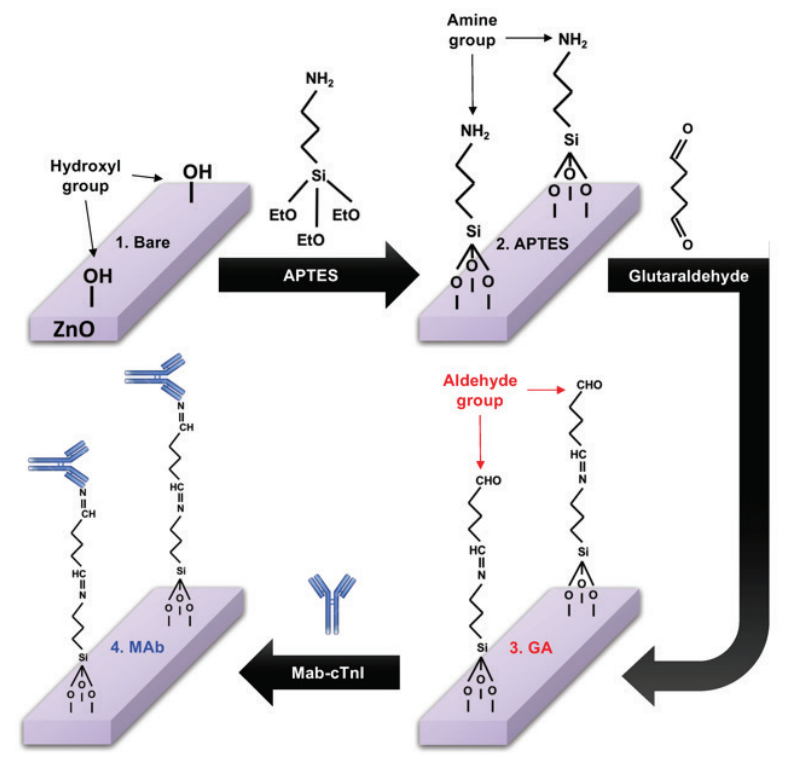

Fig. 3. Overall stages of the surface functionalization and immobilization on the $\mathrm{ZnO}-\mathrm{NPs}$ thin film [22].

\subsection{Integration with substrate-gate biasing}

Several gating approaches on FET-based biosensor have been demonstrated in the literature [23-29], which are possible to enhance their performance in biomolecules detection with higher sensitivity. Jieun Lee et al. [26] and M. Nuzaihan et al. [28] utilized a single liquid and molecular gate control, respectively, where modulation of surface charge through the nanowires (gate) occurred during interaction between bio-receptor and target molecule, thus influences the conductivity of the nanowires. In addition, different device architecture was presented by Ahn et al. [30], a double-gate nanowire FET, where the sensitivity is improved by controlling the conduction channel in the silicon nanowire (SiNW) through the application of bias symmetric/asymmetric on double-gate, which are located at both sides of the nanowire. Different approach or device architecture of double gate FET $[23,24,29,31]$ was presented utilizing the back-gate, which provides low $\mathrm{V}_{\mathrm{T}}$ values, suggests a more effective back-gate coupling.

Fathil et al. [22] demonstrated various substrate-gate voltage $\left(\mathrm{V}_{\mathrm{SG}}\right)$ biases effect on the electron concentration of the $\mathrm{ZnO}-\mathrm{FET}$-based biosensor. Fig. 4 shows the horizontal cut near (a) the BOX/substrate interface and (b) near the surface of $p-n$-p junction.

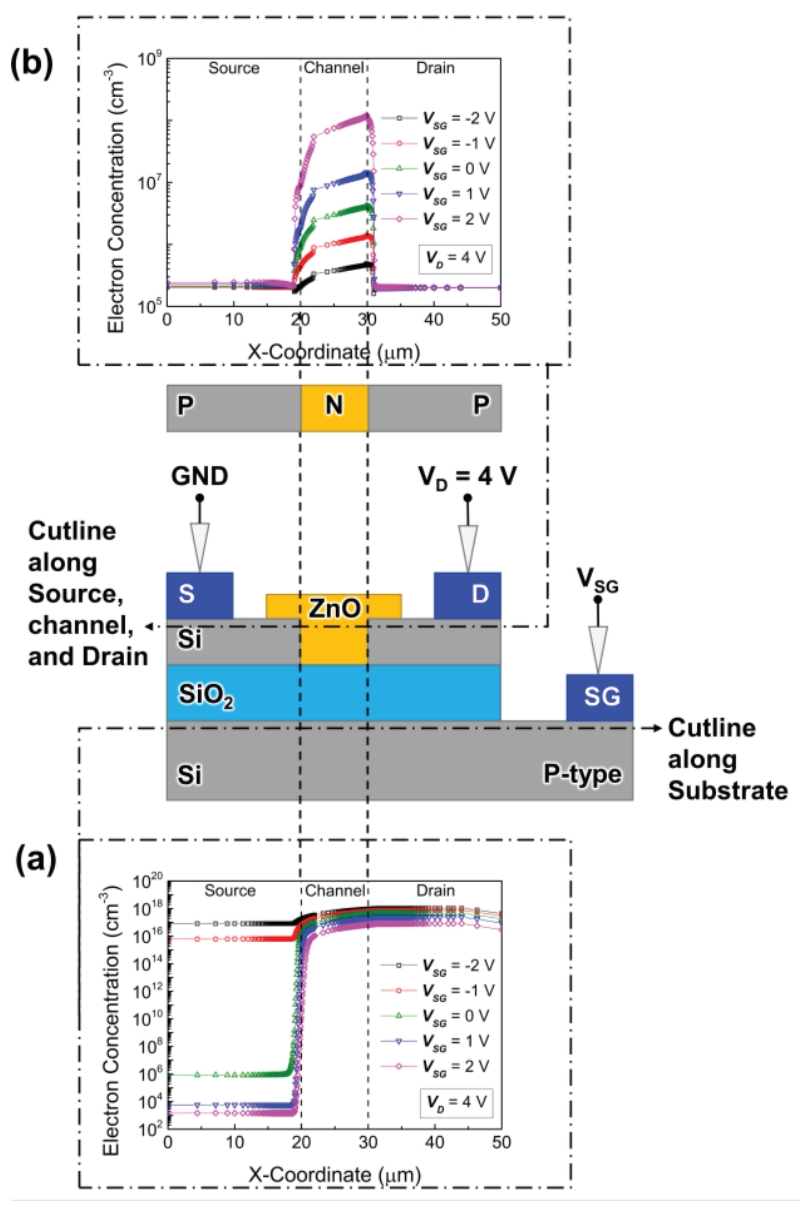

Fig. 4. Electron concentration on the FET biosensor at (a) $\mathrm{BOX} / \mathrm{Si}$ substrate interface; (b) near the surface of the source, gate and drain region for different $\mathrm{V}_{\mathrm{SG}}$ bias [22].

As shown in electron concentration cut in Fig. 4(a), at negatively $\mathrm{V}_{\mathrm{SG}}$ bias $\left(\mathrm{V}_{\mathrm{SG}}=-1\right.$ and $\left.-2 \mathrm{~V}\right)$, accumulation of high electron concentration occurs, thus introducing the inversion space charge condition in the p-type Si substrate along the BOX/Si substrate interface. This result causes the accumulation of holes, producing a hole conduction layer along the source, gate, and drain region (Fig. 4(b)), and allows more current flow between source and drain. On the contrary, as positively $\mathrm{V}_{\mathrm{SG}}$ bias $\left(\mathrm{V}_{\mathrm{SG}}=1\right.$ and $\left.2 \mathrm{~V}\right)$ are given to the substrate-gate electrode, the electron concentration along the $\mathrm{BOX} / \mathrm{Si}$ substrate interface has become lesser, especially at the location beneath the source region, hence reduced the hole concentration inside hole conduction layer at the gate region. The electrical characterization of the FET biosensors, respectively, as a function of $\mathrm{I}_{\mathrm{D}}$ with various $\mathrm{V}_{\mathrm{SG}}\left(\mathrm{V}_{\mathrm{SG}}=\right.$ - 
2, -1, 0, 1, and $2 \mathrm{~V}$ ) as in Fig. 5. The results show the biosensor exhibits a linear characteristic during forward biased, and shift of $\mathrm{V}_{\mathrm{T}}$ to the left with increases of $\mathrm{I}_{\mathrm{D}}$ for negatively $\mathrm{V}_{\mathrm{SG}}$. Opposite trend can be observed for positively $\mathrm{V}_{\mathrm{SG}}$.

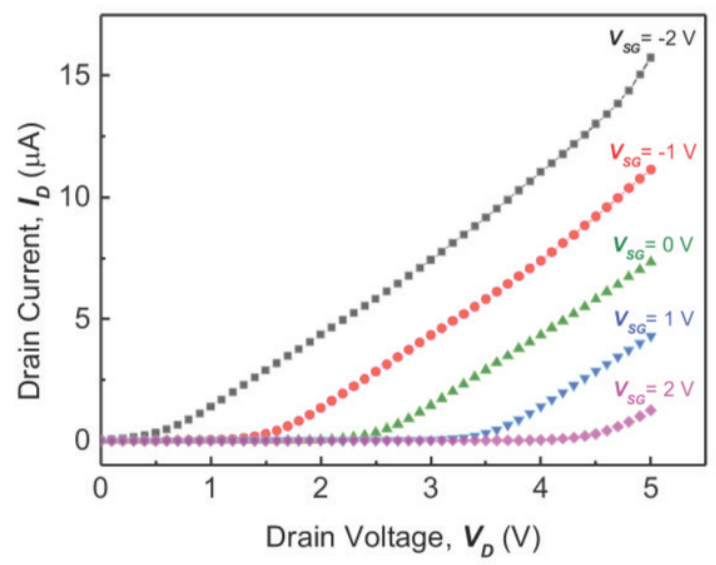

Fig. 5. Different $V_{S G}$ bias effect in term of $I_{D}$ against $V_{D}$ characteristic on the ZnO-FET biosensor with substrate-gate coupling [22].

\section{Detection of cTnl cardiac biomarker}

Fathil et al. [22] demonstrated a FET-based biosensor's example for the detection of cTnI biomarker by using zinc oxide ( $\mathrm{ZnO})$-FET biosensor. The device exhibited a significant decrease in measured $I_{D}$ as the concentrations of cTnI biomarker were increased from 1 $\mathrm{ng} / \mathrm{ml}, 10 \mathrm{ng} / \mathrm{ml}, 100 \mathrm{ng} / \mathrm{ml}, 1 \mu \mathrm{g} / \mathrm{ml}$ to $10 \mu \mathrm{g} / \mathrm{ml}$ as shown in Fig. 6.

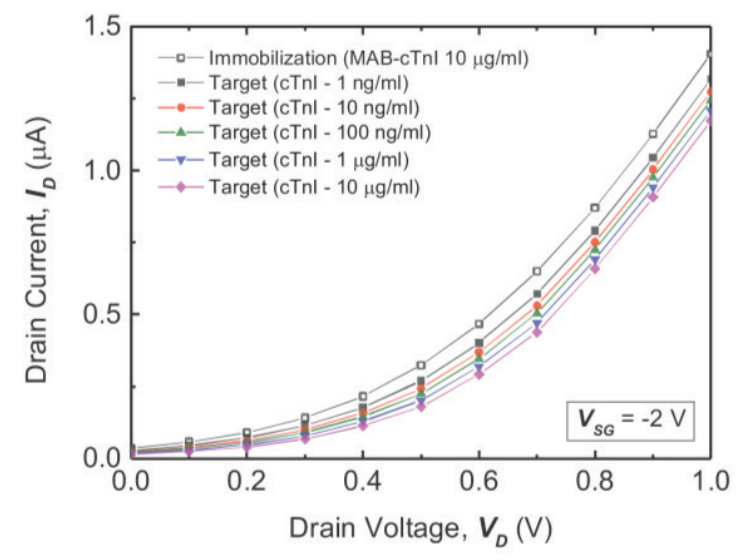

Fig. 6. ID against $V_{D}$ characteristic at different $c T n I$ biomarker concentrations.

This event occurs due to the fact that cTnI biomarkers have high positive charge (pI 9.87) [32], which repulse the positively charge, decreased the hole concentration, and thus diminished the hole conduction layer of the n-type $\mathrm{ZnO}-\mathrm{NPs}$ thin film between p-type source and drain region, hence reduced the measured $I_{D}$. The sensitivity of our biosensor [28], which is $35.3 \mathrm{nA} \cdot(\mathrm{g} / \mathrm{ml})^{-1}$ with limit of detection (LOD) of $3.24 \mathrm{pg} / \mathrm{ml}$ [22]. The specificity of the biosensor was also confirmed with $10 \mu \mathrm{g} / \mathrm{ml}$ of cTnT biomarker as non-specific protein (control), and $10 \mu \mathrm{g} / \mathrm{ml}$ of cTnI target biomarker. In comparison, a huge change in $\mathrm{I}_{\mathrm{D}}(\sim 17 \%)$ was displayed by $10 \mu \mathrm{g} / \mathrm{ml}$ of $\mathrm{cTnI}$ biomarker compared to $10 \mu \mathrm{g} / \mathrm{ml}$ cTnT noncomplementary biomarker $(\sim 1 \%)$. Only cTnI biomarker, which exhibited a specific binding to the MAb-cTnI as bio-receptor allows the relative changes in measured $I_{D}$ [22].

\section{Conclusion and future perspective}

cTnI detections in recent years through the mean of electrical label-free based biosensors have been rising due to their ability to directly interpret interactions between cTnI and the transducers' surface into readable electrical signals. FET-based biosensors, have become favourable method among researchers due to ultra-sensitivity and high specificity, which are most likely reduce falsenegative results in terms of AMI determination and gives more accurate cTnI levels in real time. Simplicity in the detection of cTnI without the need for labels addition has enabled a simple and inexpensive fabrication process, thus reducing the complexity of the device detection. Although with all the promising privileges presented by the electrical label-free detection biosensors, they are not intended as a substitute for the existing technologies for AMI detection. Rather, the biosensors should be used side by side with the present methods, such as ECG, to aid and offer quantitative outcomes for the determination of cardiac biomarker levels in patients; thus, allowing doctors to justify their decision making in the diagnostics of AMI with accurately, quickly, and wisely.

With the rapid advancement of technology, there is hope that, in the future, an optimized, stable and portable biosensor can be fabricated as a plug-in accessory to a smartphone or tablet that serves as a cTn level reader; thus, enabling the diagnosis process to be performed anywhere and at any time as in Fig. 7.

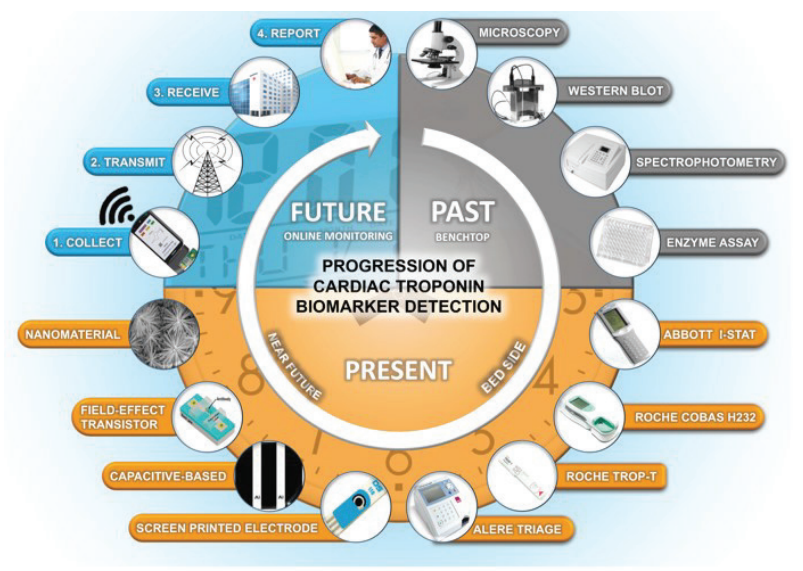

Fig. 7. Progression of cTn biomarkers devices detection from the past, present, and future perspective, a transition from benchtop towards translational research [33].

The results could not only be displayed to the user but also transmitted to the hospital for further analysis by physicians. For the patient, this will save significant travelling and waiting time at the hospital for a medical 
check-up, while from the doctors' point of view, these tests could reduce the number of patients with this condition falling under false-negative trips to the emergency department. Based on the results, doctors can provide suitable advice to the patient through voice conversation via smartphone depending on the level of cTn in the patient's blood or can even dispatch an ambulance to the patient's present location if the cTn level is above the reference value at an early stage. Hence, the probability to save lives due to MI can be increased significantly.

The authors are grateful to the Department of Higher Education, Ministry of Higher Education, (KPT) for funding this research through the Fundamental Research Grant Scheme (FRGS) with the grant number 9003-00536. The authors also would like to acknowledge all the team members at the Institute of Nano Electronic Engineering (INEE), Universiti Malaysia Perlis (UniMAP), for their guidance and help.

\section{References}

1. S. B. Rosalki, R. Roberts, H. A. Katus, E. Giannitsis, and J. H. Ladenson, Clin. Chem. 50, 2205 (2004)

2. A. S. Jaffe and J. Ordonez-Llanos, Rev. Española Cardiol. (English Ed. 63, 763 (2010)

3. E. Burcu Bahadır and M. Kemal Sezgintürk, Talanta 132, 162 (2015)

4. R. S. Wright, J. L. Anderson, C. D. Adams, C. R. Bridges, D. E. Casey, S. M. Ettinger, F. M. Fesmire, T. G. Ganiats, H. Jneid, A. M. Lincoff, E. D. Peterson, G. J. Philippides, P. Theroux, N. K. Wenger, J. P. Zidar, and A. K. Jacobs, Circulation 123, 2022 (2011)

5. S. Agewall, E. Giannitsis, T. Jernberg, and H. Katus, Eur. Heart J. 32, 404 (2011)

6. M. Mascini and S. Tombelli, Biomarkers 13, 637 (2008)

7. A. Qureshi, Y. Gurbuz, and J. H. Niazi, Sensors Actuators B Chem. 171-172, 62 (2012)

8. T. Kong, R. Su, B. Zhang, Q. Zhang, and G. Cheng, Biosens. Bioelectron. 34, 267 (2012)

9. P. Estrela, A. G. Stewart, F. Yan, and P. Migliorato, Electrochim. Acta 50, 4995 (2005)

10. G. Zhang and Y. Ning, Anal. Chim. Acta 749, 1 (2012)

11. D. Sarkar, W. Liu, X. Xie, A. C. Anselmo, S. Mitragotri, and K. Banerjee, ACS Nano 8, 3992 (2014)

12. U. Brand, L. Brandes, V. Koch, T. Kullik, B. Reinhardt, F. Rüther, T. Scheper, K. Schügerl, S. Wang, X. Wu, R. Ferretti, S. Prasad, and D. Wilhelm, Appl. Microbiol. Biotechnol. 36, 167 (1991)

13. S. Liu and X. Guo, NPG Asia Mater. 4, e23 (2012)

14. P. Bergveld, Sensors Actuators B Chem. 88, 1 (2003)

15. P. R. Solanki, A. Kaushik, V. V. Agrawal, and B. D.
Malhotra, NPG Asia Mater. 3, 17 (2011)

16. Q. Xu, C. Mao, N. Liu, J. Zhu, and J. Sheng, Biosens. Bioelectron. 22, 768 (2006)

17. S. P. Singh, S. K. Arya, P. Pandey, B. D. Malhotra, S. Saha, K. Sreenivas, and V. Gupta, Appl. Phys. Lett. 91, 63901 (2007)

18. A. F. Collings and F. Caruso, Reports Prog. Phys. 60, 1397 (1997)

19. R. Monošík, M. Stred'anský, and E. Šturdík, Acta Chim. Slovaca 5, 109 (2012)

20. A. Chaubey and B. D. Malhotra, Biosens. Bioelectron. 17, 441 (2002)

21. J.-Y. Yoon, Introduction to Biosensors (Springer International Publishing, Cham, 2016)

22. M. F. M. Fathil, M. K. Md Arshad, A. R. Ruslinda, S. C. B. Gopinath, M. Nuzaihan M.N., R. Adzhri, U. Hashim, and H. Y. Lam, Sensors Actuators B Chem. 242, 1142 (2017)

23. E. Buitrago, M. F.-B. Badia, Y. M. Georgiev, R. Yu, O. Lotty, J. D. Holmes, A. M. Nightingale, H. M. Guerin, and A. M. Ionescu, Sensors Actuators B Chem. 199, 291 (2014)

24. P. Dai, A. Gao, N. Lu, T. Li, and Y. Wang, Jpn. J. Appl. Phys. 52, 121301 (2013)

25. Z. Iskierko, M. Sosnowska, P. S. Sharma, T. Benincori, F. D’Souza, I. Kaminska, K. Fronc, and K. Noworyta, Biosens. Bioelectron. 74, 526 (2015)

26. J. Lee, J. Jang, B. Choi, J. Yoon, J.-Y. Kim, Y.-K. Choi, D. Myong Kim, D. Hwan Kim, and S. Choi, Sci. Rep. 5, 12286 (2015)

27. J. Li, M. Sun, X. Wei, L. Zhang, and Y. Zhang, Biosens. Bioelectron. 74, 423 (2015)

28. M. N. M. Nuzaihan, U. Hashim, M. K. Md Arshad, S. R. Kasjoo, S. F. A. Rahman, A. . Ruslinda, M. F. M. Fathil, R. Adzhri, and M. M. Shahimin, Biosens. Bioelectron. 83, 106 (2016)

29. J.-K. Park, H. Jang, J. Park, and W. Cho, Solid. State. Electron. 97, 2 (2014)

30. J.-H. Ahn, S.-J. Choi, J.-W. Han, T. J. Park, S. Y. Lee, and Y.-K. Choi, Nano Lett. 10, 2934 (2010)

31. P. G. Fernandes, R. A. Chapman, O. Seitz, H. J. Stiegler, H.-C. Wen, Y. J. Chabal, and E. M. Vogel, IEEE Electron Device Lett. 33, 447 (2012)

32. S. Eriksson, Clin. Chem. 49, 1095 (2003)

33. M. F. M. Fathil, M. K. Md Arshad, A. R. Ruslinda, M. Nuzaihan, S. C. B. Gopinath, R. Adzhri, and U. Hashim, Anal. Chim. Acta (2016) 